\title{
12 Krankheiten der männlichen Brust
}

\author{
Hans-Christian Schuppe und Jens W. Jacobeit
}

\subsection{Einleitung}

Diagnostik und Therapie von Krankheiten der Brustdrüsen und umgebender Strukturen beim Mann gehören zu den Aufgabengebieten der Andrologie, die Untersuchung der männlichen Brust sollte jedoch auch im Rahmen der allgemeinärztlichen oder internistischen Versorgung ausreichende Beachtung finden.

Zu dem Organkomplex der Brust gehören die pigmentierte Brustwarze mit freien Talgdrüsen, der pigmentierte und behaarte Warzenhof mit apokrinen Drüsen sowie der Drüsenkörper, der in den verschiedenen Altersabschnitten hormonell gesteuert Wachstums- und Ruhephasen unterliegt (Schreiber et al. 2005). Bei der häufig vorkommenden Vergrößerung der Brust bzw. des Brustdrüsenkörpers, der Gynäkomastie, suchen junge Männer vor allem aufgrund des belastenden körperlichen Erscheinungsbildes ärztlichen Rat, ältere Männer dagegen aus der Sorge heraus, eine bösartige Erkrankung zu haben (Khan u. Blamey 2003). In der Tat stellt das virile Mammakarzinom die wichtigste Differenzialdiagnose der Gynäkomastie dar. Mamille und Areola können andererseits Manifestationsort entzündlicher Dermatosen sowie benigner und maligner Tumoren der Haut und Hautanhangsgebilde sein. Schließlich sind als harmlose Fehlbildungen überzählige Mamillen (Polythelie) und Drüsenkörper (Polymastie) abzugrenzen, die mit einer Häufigkeit von 1-2\% bei beiden Geschlechtern auftreten (Bork 1995). Sie finden sich entweder als „akzessorisches“ Brustgewebe auf der sog. Milchleiste, dem embryonalen Entstehungsort, oder versprengt „aberrierend“. 


\subsection{Gynäkomastie}

\subsubsection{Definition}

Bei der Gynäkomastie handelt es sich um eine tastbare, uni- oder bilaterale, in einigen Fällen schmerzhafte Vergrößerung des männlichen Brustdrüsenkörpers. Von der sog. echten Gynäkomastie wird die Vergrößerung der männlichen Brust durch eine Vermehrung des Fettgewebes (Lipomastie) oder einen benignen Tumor als Pseudogynäkomastie abgegrenzt (Schreiber et al. 2005; Braunstein 2007). Aufgrund der klinisch oft schwierigen Differenzierung, insbesondere zwischen echter Gynäkomastie und Lipomastie, wird von einigen Autoren die Vergrößerung der männlichen Brust insgesamt als Gynäkomastie bezeichnet und ein „lump type“ (umschriebener retro-areolärer Knoten) von einem „fatty type“ (diffuse Vermehrung des Fettgewebes) unterschieden (Khan u. Blamey 2003; Krause 2006).

\subsection{2 Ätiopathogenese}

Wachstum und Differenzierung des Brustdrüsengewebes beim Mann wird durch Östradiol über Östrogenrezeptoren stimuliert, während Testosteron über den Androgenrezeptor eine inhibierende Wirkung entfaltet (Mathur u. Braunstein 1997). Der Gynäkomastie als klinischem Leitsymptom liegt somit eine gestörte Balance zwischen Androgen- und Östrogenangebot bzw. -wirkung zugrunde, die vielfältige Ursachen haben kann (Carlson 2011; s. Tab. 1 u. 2). Darüber hinaus werden lokale Faktoren wie z.B. hypersensitives Brustgewebe beschrieben.

\section{Tab. 1 Pathophysiologie der Gynäkomastie}

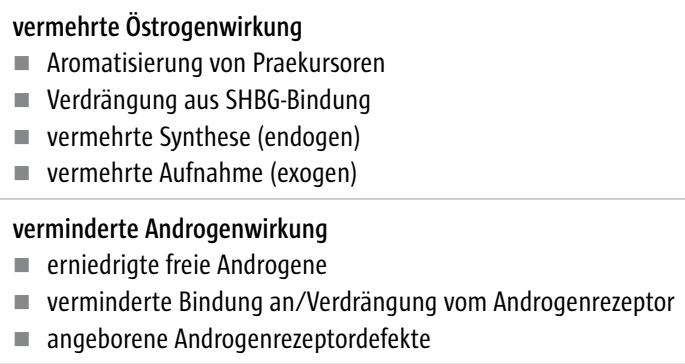

hypersensitives Brustgewebe

In bestimmten Lebensabschnitten - im Neugeborenenalter, in der Pubertät und im Senium - ist die Gynäkomastie als physiologisch anzusehen. Bei ca. der Hälfte der Patienten mit einer pathologischen Gynäkomastie bleibt die Ursache unklar (idiopathische Gynäkomastie).

Die Gynäkomastie ist ein Leitsymptom mit vielfältigen Ursachen. 
Tab. 2 Pathologische Formen der Gynäkomastie

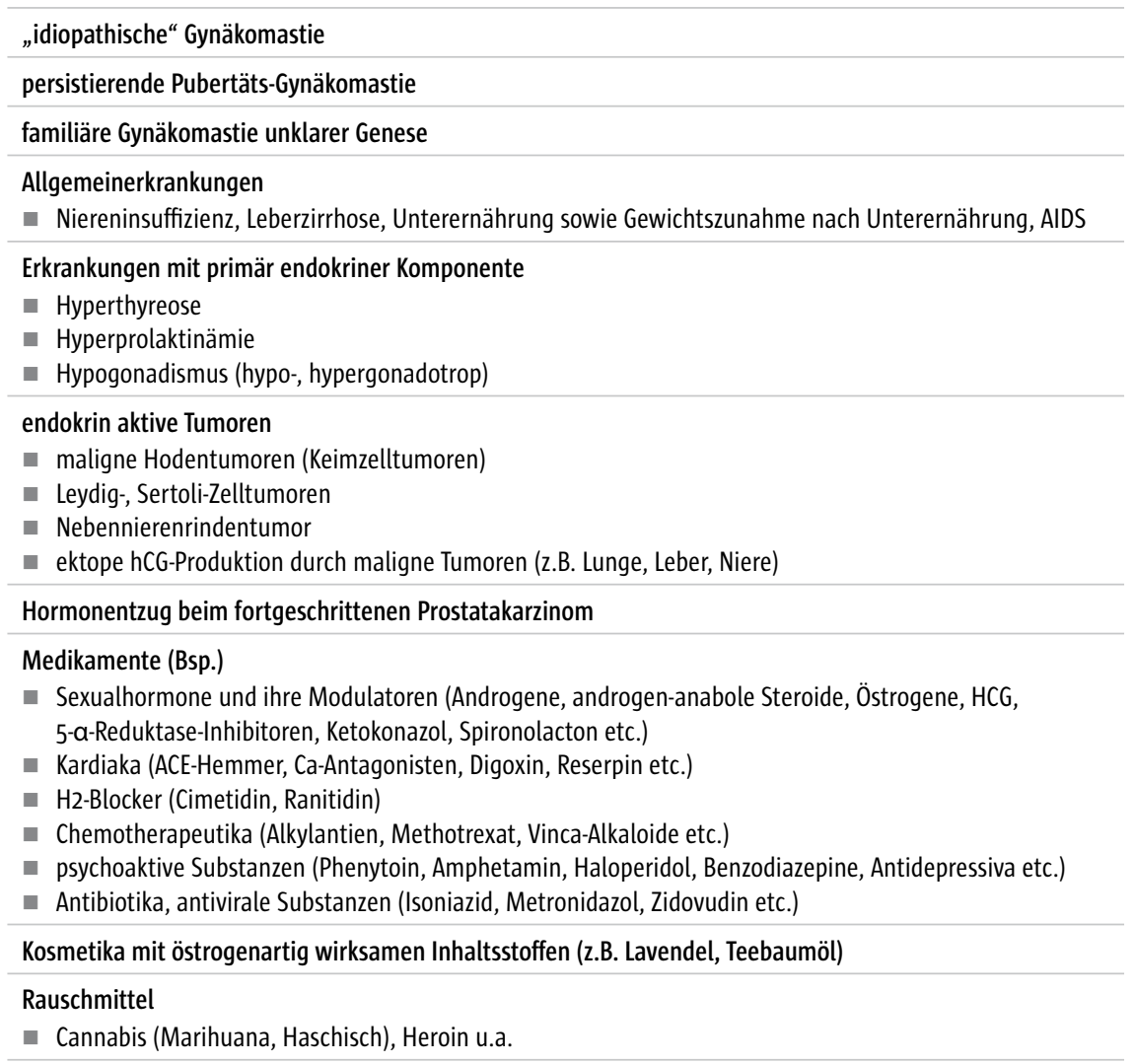

\subsubsection{Prävalenz}

Die Häufigkeitsangaben zu verschiedenen Formen der Gynäkomastie differieren stark, möglicherweise vor dem Hintergrund methodischer Probleme in der Erfassung und Klassifikation (Schreiber et al. 2005). Im Neugeborenenalter findet man bei bis zu 90\% der Jungen eine harmlose und rasch reversible Gynäkomastie, die Prävalenz in der Pubertät wird mit 40-70\% angegeben (Kauf 1998; Kumanov et al. 2007). In der erwachsenen männlichen Bevölkerung wird eine Häufigkeit von 30-60\% berichtet, wobei im Senium mit der höchsten Prävalenz zu rechnen ist (Niewöhner u. Nuttal 1984; Braunstein 2007).

\subsubsection{Klinik und Diagnostik}

Zur Objektivierung einer Gynäkomastie wurden verschiedene Maße vorgeschlagen:

- Die im Palpationsbefund zu erhebende Dicke einer horizontalen Hautfalte unter Einschluss der Brustwarze (pathologisch $>2 \mathrm{~cm}$, bei adipösen Männern $>3 \mathrm{~cm}$ ),

- alternativ der Durchmesser des Brustwarzenhofes (pathologisch $>3 \mathrm{~cm}$ ) (Krause 2006). 


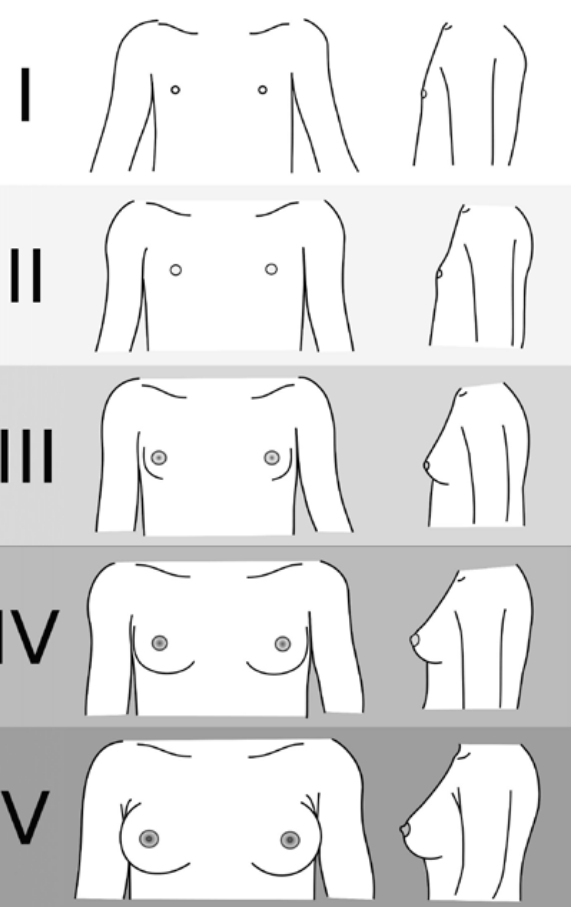

Abb. 1 Stadieneinteilung der Gynäkomastie, I = kein Drüsenkörper tastbar, II = Warzenhof vergrößert, Drüse vorgewölbt, III = Drüsenkörper größer als Warzenhof, IV = solider Drüsenkörper, $V$ = entspricht weiblicher Brust (Abbildung nach Michal Komorniczak (Polen); Creative Commons-Lizenz Namensnennung-Weitergabe unter gleichen Bedingungen 3.0 Unported, http://creativecommons.org/licenses/by-sa/3.0/deed.de)

Die genannten Werte sind allerdings weder als klinische „Thresholds“ allgemein akzeptiert noch altersdifferenziert. Eine qualitative Stadieneinteilung der Brustvergrößerung kann in der Praxis sehr einfach anhand der eigentlich zur Beurteilung der Pubertätsentwicklung bei Mädchen etablierten Tanner-Stadien erfolgen (s. Abb. 1).

Klinisch imponiert die Gynäkomastie als ein- oder beidseitige Vergrößerung der Brustwarze und des Warzenhofes einschließlich eines in Strängen tastbaren, z.T. dolenten Drüsenkörpers (s. Abb. 2a-d). Das zunächst proliferative Brustdrüsenwachstum (reversibel) kann im Verlauf in ein Stadium der Fibrosierung (irreversibel) übergehen, was in der Regel nach einer Bestandsdauer von mehr als einem Jahr geschieht.

Die wichtigste Differenzialdiagnose einer Gynäkomastie, insbesondere bei der einseitigen Lokalisation, ist das Mammakarzinom. 

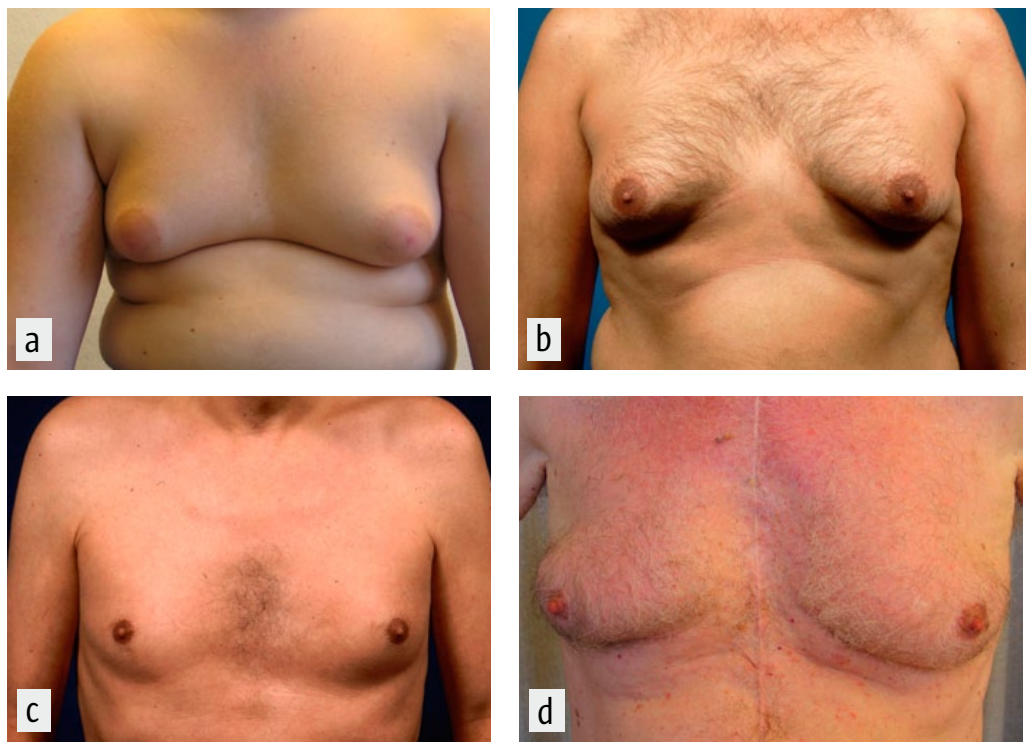

Abb. 2a-d Klinik der Gynäkomastie, a: pubertätsassoziierte Gynäkomastie bei 16-jährigem Jugendlichen mit Adipositas; b: idiopathische Gynäkomastie bei 36-jährigem Mann; c: diskrete unilaterale Gynäkomastie links bei Leydig-Zelltumor, 45-jähriger Patient; d: medikamentös induzierte Gynäkomastie bei 82-jährigem Patienten mit koronarer Herzkrankheit (Einnahme von Spironolacton)

Abzugrenzen sind darüber hinaus:

- Fibrosen, Zysten

- Entzündungsreaktionen

- Traumata mit Hämatombildung

- Tumoren und Metastasen

- venöse oder lymphatische Abflussstörungen (Karzinome benachbarter Organe, Struma, Lymphome)

Das diagnostische Vorgehen bei Gynäkomastie sollte individuell nach der speziellen Anamnese und richtungweisenden Befunden abgestimmt werden (Schreiber et al. 2005; Braunstein 2007; Carlson 2011). Eine schmerzfreie, bereits länger bestehende, eher als Zufallsbefund entdeckte, evtl. im typischen Alter auftretende oder sicher als persistierendes pubertäts-assoziiertes Geschehen einzuordnende Gynäkomastie macht eine intensive Diagnostik nicht zwingend erforderlich. Die abgestufte Diagnostik bei pathologischen Formen der Gynäkomastie ist in Tabelle 3 dargestellt.

In der bildgebenden Diagnostik hat die sonografische Darstellung der Gynäkomastie Priorität: Es finden sich weitgehend uncharakteristische Befunde, allerdings lassen sich der Drüsenkörper vom Fettgewebe abgrenzen und evtl. Zysten oder Verkalkungen erfassen. Aussagen zur Dignität sind nicht immer möglich. Eine Mammografie ist im Verdachtsfall auf einen malignen Tumor oder bei sonstigen sonografisch unklaren Befunden angezeigt. Zur Beurteilung der Dignität in der bildgebenden Diagnostik kann das vom American College of Radiology (2003) empfohlene System BI-RADS ${ }^{\text {TM }}$ (Breast Imaging - Reporting And Data System) herangezogen werden (s. Tab. 4). 


\section{Tab. 3 Diagnostisches Vorgehen bei Gynäkomastie}

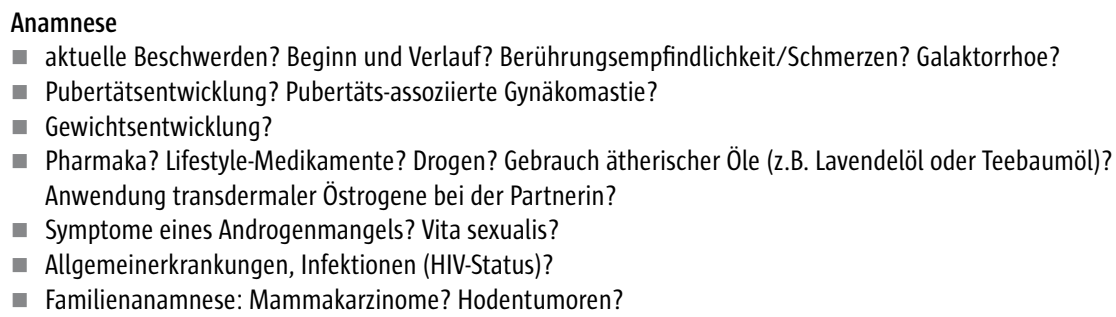

Klinik

- Allgemeinstatus, Lokalbefund (möglichst Fotodokumentation), akzessorisches/aberrierendes Brustgewebe, andrologischer Genitalbefund, Lymphknotenstatus

\section{bildgebende Diagnostik}

- Mamma-Sonografie (mind. 7,5 MHz Schallkopf); Hoden-Sonografie!

- Optional: Mammografie, Röntgen-Thorax, MRT Hypophyse, Sonografie/CT Nebennieren, Leber

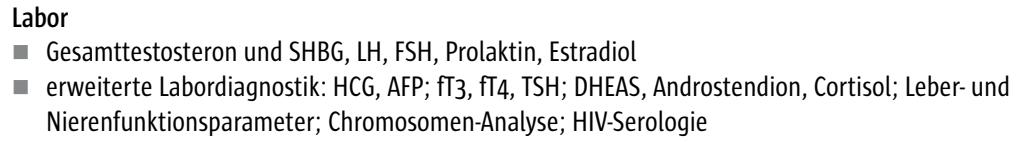

histologische Klärung bei V.a. Neoplasie

\section{Tab. 4 BI-RADS ${ }^{\mathrm{TM}}$ (Breast Imaging-Reporting And Data System): Kategorien der Dignitätsbeurteilung mammografischer, sonografischer und MRT-Befunde}

\begin{tabular}{|ll|}
\hline 0 & Untersuchung unvollständig \\
\hline 1 & unauffällig \\
\hline 2 & gutartig \\
\hline 3 & unklar - eher gutartig - kontrollbedürftig \\
\hline 4 & unklar - suspekt - abklärungsbedürftig \\
\hline $4 a$ & wenig suspekt \\
\hline $4 b$ & intermediär \\
\hline $4 C$ & höhergradig suspekt \\
\hline 5 & karzinomverdächtig \\
\hline 6 & gesichertes Karzinom \\
\hline
\end{tabular}

\section{Palpation und Sonografie des Skrotalinhalts zum Ausschluss eines Hoden- tumors sind bei der Abklärung einer Gynäkomastie obligat.}




\subsubsection{Therapie}

\section{Bei der Therapieplanung ist zu berücksichtigen, dass nicht jede Gynäkomastie behandelt werden muss. Insbesondere während der Pubertät ist zunächst ein abwartendes Verhalten angezeigt, da mit einer hohen Spontanremissionsrate zu rechnen ist.}

Die Behandlung der pathologischen Formen einer Gynäkomastie zielt - sofern möglich - auf die Ausschaltung kausaler Faktoren in Abhängigkeit vom Ergebnis der Diagnostik ab:

- Medikamente

- Drogen

- Behandlung eines endokrinen Hypogonadismus

- Therapie eines Leydigzelltumors (selten) oder

- eines malignen Keimzelltumors des Hodens.

Die Therapie der idiopathischen Gynäkomastie erfolgt in Abhängigkeit der Schmerzsymptomatik und des Leidensdrucks des Patienten. Ein medikamentöser Therapieversuch ist vor allem in der proliferativen Phase zu erwägen (siehe Übersicht bei Schreiber et al. 2005).

Eine zugelassene medikamentöse Therapie der Gynäkomastie gibt es in Deutschland nicht. Studiendaten liegen vor allem für Tamoxifen vor. Das Präparat führte zu kompletten oder zumindest partiellen Remissionen und Schmerzfreiheit (in der Praxis „off-label“ als Heilversuch, [10-]20 mg/über 3[-6] Monate). Für alternative Behandlungsansätze wie z.B. mit Clomifencitrat, Danazol, den Aromatasehemmern Testolacton und Anastrazol oder auch die lokale Applikation von Dihydrotestosteron ist die Datenbasis sehr begrenzt.

Bei länger als einem Jahr bestehender bereits fibrosierter Veränderung und entsprechendem Leidensdruck des Patienten besteht die Indikation für eine subkutane Mastektomie und Liposuktion durch einen erfahrenen plastischen Chirurgen oder Senologen (Fruhstorfer u. Malata 2003; Petty et al. 2010). Zuvor muss die Klärung der Kostenübernahme erfolgen. Hier empfiehlt es sich, nach oben beschriebener Diagnostik und Befunddokumentation einen entsprechenden Bericht an den Medizinischen Dienst der zuständigen Krankenversicherung zu formulieren.

Eine Sonderstellung nimmt die Behandlung der Gynäkomastie ein, die mit der therapeutischen Androgen-Deprivation bei fortgeschrittenem Prostata-Karzinom auftritt. Zum Einsatz kommen prophylatische Radiatio sowie Aromatasehemmer oder Tamoxifen (Dobs u. Darkes 2005; Carlson 2011). Hierbei scheint die tägliche Gabe von Tamoxifen die beste Wirkung zu entfalten (Bedognetti et al. 2010).

\subsection{Mammakarzinom}

Männlicher Brustkrebs ist selten, stellt jedoch insbesondere bei einseitigen Befunden die wichtigste Differenzialdiagnose einer Gynäkomastie dar. Die Häufigkeit weist 
sowohl regionale als auch ethnisch bedingte Unterschiede auf, für Europa wird mit einer Inzidenz von ca. 1/100.ooo pro Jahr gerechnet (Backe 2002; Fentiman et al. 2006). Daten aus den USA belegen einen Anstieg der Inzidenz des virilen Mammakarzinoms, das ca. $1 \%$ aller Brustkrebsfälle ausmacht, um über $25 \%$ in den letzten 25 Jahren (Giordano et al. 2004). Der Altersgipfel der Erkrankung liegt bei ca. 70 Jahren, wobei der Tumor oft erst im fortgeschrittenen Stadium festgestellt wird und damit eine schlechte Prognose hat (5-Jahres-Überlebensrate 40-65\%).

\section{Die gezielte Anamnese kann Hinweise auf eine genetische Disposition geben.}

Ca. 20\% der betroffenen Männer haben Verwandte ersten Grades, die ebenfalls an einem Mammakarzinom erkrankt sind. Autosomal dominant vererbbare Mutationen finden sich vor allem in den BRCA1- und -2-Genen, wobei BRCA-2-Genmutationen mit einem höheren Risiko assoziiert sind (Backe 2002; Fentiman et al. 2006). Bereits bei jungen Männern muss mit einer Erkrankung gerechnet werden. Weiterhin gehen Androgenresistenzsyndrome, erbliche kolorektale Karzinome, das Cowden-Syndrom oder die familiäre Häufung von Ovarialkarzinomen mit einem erhöhten Risiko für ein viriles Mammakarzinom einher. Die Ergebnisse einer Reihe epidemiologischer Studien weisen darauf hin, dass auch Patienten mit einem Klinefelter-Syndrom ein 20-50-fach höheres Risiko tragen, an einem Mammakarzinom zu erkranken (Brinton 2011). Inwieweit die Gynäkomastie an sich mit einem mäßig erhöhten Mammakarzinom-Risiko einhergeht, wird kontrovers diskutiert (Fentiman et al. 20o6; Brinton et al. 2010). Am Beispiel einer Finasterid-induzierten Gynäkomastie wurde andererseits auf die Gefahr der Verschleierung eines virilen Mammakarzinoms hingewiesen (Arzneimittelkommission der deutschen Ärzteschaft 2008).

Klinisch sind neben dem suspekten Palpationsbefund mit einem derben, meist schmerzlosen subareolären Infiltrat die Einbeziehung der Haut mit Retraktionen und „Apfelsinenschalenphänomen“ sowie eine sero-sanguinöse Sekretion aus der Mamille wichtige Zeichen eines Mammakarzinoms, oft ist bei Diagnosestellung bereits eine axilläre Lymphknotenschwellung nachweisbar, in späteren Stadien treten ulzerierende Knoten auf (Fentiman et al. 2006). Der häufigste Tumortyp ist das invasiv-duktale Mammakarzinom. Ekzem-ähnliche Veränderungen der Areola infolge der epidermalen Ausbreitung eines intraduktalen Karzinoms (M. Paget) sind beim Mann allerdings selten. Ebenso wie beim weiblichen Mammakarzinom kann es zu einer regionalen Ausbreitung mit lentikulären Hautmetastasen sowie Erysipelas carcinomatosum (flächenhafte Entzündung und Infiltraion der Haut infolge des Befalls der Lymphgefäße) kommen.

In allen Verdachtsfällen ist die Durchführung einer Mammasonografie und Mammografie zu fordern, das weitere Procedere umfasst neben der histologischen Abklärung die bildgebende Diagnostik zum Ausschluss einer Metastasierung. Die Therapie umfasst ebenso wie bei der Frau chirurgische Maßnahmen (Mastektomie, ggf. axilläre Lymphknoten-Dissektion), Radiatio, adjuvante Chemotherapie sowie die Gabe von Antiöstrogenen bei rezeptorpositiven Tumoren (Fentiman et al. 2006). 


\subsection{Hautkrankheiten}

\subsubsection{Entzündliche Dermatosen}

Mamille und Areola sind Manifestationsort entzündlicher Dermatosen, insbesondere bei disseminiertem bzw. generalisiertem Befall des Integuments (Bork 1995). Als lokalisierte Entzündungsreaktion tritt das Mamillenekzem mit Juckreiz, Rötung, Erosionen und Schwellung auf, nicht selten im Verlauf chronisch-rezidivierend. Zumeist liegt eine atopische Dermatitis zugrunde, aber auch allergische Kontaktreaktionen können Mamille und Areola betreffen. Als sehr seltene Differenzialdiagnose ist ein M. Paget zu beachten (s. Kap. II.12.3). Iatrogene Formen des Mamillenekzems bzw. einer Mastitis treten infolge von Brustwarzen-Piercings, Tätowierungen oder anderen Manipulationen auf.

Von der Gynäkomastie differenzialdiagnostisch abzugrenzen ist die Lymphadenosis cutis benigna, ein B-Zell-Pseudolymphom der Haut. Häufig stellt eine Infektion mit Borrelia burgdorferi nach Zeckenstich die Ursache dar (Borrelienlymphozytom; Gissler u. Heiniger 2002), aber auch Stiche anderer Insekten, Impfungen oder die o.g. traumatischen Faktoren kommen als Auslöser in Betracht.

Eine sog. nävoide Hyperkeratose kann z.B. im Rahmen von Ichthyosen auftreten; es finden sich Hyperkeratosen mit Hyperpigmentierung uni- oder meist bilateral an den Mamillen, die auf Areolae und umliegende Haut übergreifen können (Kubota et al. 2000).

\subsubsection{Tumoren der Haut und Hautanhangsgebilde}

Eine seltene, nicht therapiebedürftige Veränderung stellt die Talgdrüsenhyperplasie der Areola dar (Krisp u. Krause 2003). Darüber hinaus können zahlreiche benigne und maligne Tumoren der Haut und Hautanhangsgebilde im Bereich der männlichen Brust auftreten. Zu den wichtigsten epithelialen Tumoren zählen harmlose seborrhoische Keratosen, epidermale Nävi, aber auch Basalzellkarzinome. Bei den melanozytären Tumoren sind benigne Nävuszellnävi vom malignen Melanom abzugrenzen. Schließlich treten auch im Bereich der Brust Fibrome und andere mesenchymale oder gefäßassoziierte Tumoren auf (Bork 1995). Stets ist das Mammakarzinom als Differenzialdiagnose zu beachten .

\section{Literatur}

American College of Radiology (2003) BI-RADS Mammography, Fourth Edition. http://www.acr.org/SecondaryMainMenuCategories/quality_safety/BIRADSAtlas/BIRADSAtlasexcerptedtext/BIRADSMammographyFourthEdition.aspx, abgerufen am 28.09.2011

Arzneimittelkommission der deutschen Ärzteschaft (2008) Eine Gynäkomastie durch Finasterid kann die Diagnose eines Mammakarzinoms beim Mann verzögern. Dt. Ärzteblatt 105, B2110

Backe I (2002) Brustkrebs des Mannes Dt. Ärzteblatt 99, B970-B974

Bedognetti D, Rubagotti A, Conti G, Francesca F, De Cobelli 0, Canclini L, Gallucci M, Aragona F, Di Tonno P, Cortellini P, Martorana G, Lapini A, Boccardo F (2010) An open, randomized, multicentre, phase 3 trial comparing the efficacy of two tamoxifen schedules in preventing gynaecomastia induced by bicalutamide monotherapy in prostate cancer patients. Eur Urol 57, 238-245 
Bork K (1995) Haut und Brust. Dermatologische Aspekte der Brustkrankheiten. Fischer-Verlag; Stuttgart, Jena, New York

Braunstein GD (2007) Clinical practice. Gynecomastia. N Engl I Med 357, 1229-1237

Brinton LA (2011) Breast cancer risk among patients with Klinefelter syndrome. Acta Pædiatrica 100, 814-818

Brinton LA, Carreon ID, Gierach GL, McGlynn KA, Gridley G (2010) Etiologic factors for male breast cancer in the U.S. Veterans Affairs medical care system database. Breast Cancer Res Treat 119, 185-192

Carlson HE (2011) Approach to the Patient with Gynecomastia. I Clin Endocrinol Metab 96, 15-21

Dobs A, Darkes M) (2005) Incidence and management of gynecomastia in men treated for prostate cancer. I Urol 174, 1737-1742

Fentiman IS, Fourquet A, Hortobagyi GN (2006) Male breast cancer. Lancet 367, 595-604

Fruhstorfer BH, Malata CM (2003) A systematic approach to the surgical treatment of gynaecomastia. Br J Plast Surg 56, 237-246

Giordano SH, Cohen DS, Buzdar AU, Perkins G, Hortobagyi GN (2004) Breast carcinoma in men: a population-based study. Cancer 101, 51-57

Gissler S, Heininger U (2002) Borrelia lymphocytoma („lymphadenosis benigna cutis“). Arch Dis Child 87, 12

Kauf E (1998) Gynäkomastie im Kindesalter. Fortschr Med 116, 23-26

Khan HN, Blamey RW (2003) Endocrine treatment of physiological gynaecomastia. BMI 327, 301-302

Krause W (2006) Gynrcomastia and benign breast hyperplasia including iatrogenic causes. In: Schill WB, Comhaire F, Hargreave TB (eds.) Andrology for the Clinician, Springer, Heidelberg, S. 225-232

Krisp A, Krause W (2003) Areolar sebaceous hyperplasia. Acta Derm Venereol 83, 61-62

Kubota Y, Koga T, Nakayama I, Kiryu $\mathrm{H}$ (2000) Naevoid hyperkeratosis of the nipple and areola in a man. $\mathrm{Br}$ J Dermatol 142, 382-384

Kumanov P, Deepinder F, Robeva R, Tomova A, Li I, Agarwal A (2007) Relationship of adolescent gynecomastia with varicocele and somatometric parameters: a cross-sectional study in 6200 healthy boys. I Adolesc Health 41, $126-131$

Mathur R, Braunstein GD (1997) Gynecomastia: pathomechanisms and treatment strategies. Horm Res 48, 95-102 Niewoehner CB, Nuttal FQ (1984) Gynecomastia in a hospitalized male population. Am I Med 77, 633-638

Petty PM, Solomon M, Buchel EW, Tran NV (2010) Gynecomastia: Evolving Paradigm of Management and Comparison of Techniques. Plast Reconstr Surg 125, 1301-1308

Schreiber G, Schanz S, Köhn FM (2005) Leitlinie - Gynäkomastie. I Dtsch Dermatol Ges 3, 561-565

Tanner IM (1962) Growth at adolescence. Blackwell, Oxford 